\title{
Lost In Transition: A young adult cancer survivor's perspective on life after cancer
}

\section{Timothy W. Buckland}

\author{
University of Alberta
}

\section{Introduction - Fitting Into An Unfamiliar Place}

From a patient's perspective, cancer is not only a physical illness. The emotional toll incurred upon diagnosis can be just as devastating and is often overlooked. As a cancer survivor, I know that for young adults this emotional hardship could not come at a worse time. A common theme of all young adults is the transitional nature of our lives. This transition represents moving from the security of adolescence to the independent development of careers and families. ${ }^{[1]}$ This development produces a fast-paced lifestyle that if interrupted by a cancer diagnosis creates a void between this fast-paced life and a stalled cancer life. For example, my diagnosis came before I was supposed to start university and led to me missing my first semester. Seven years later, upon completion of my treatment and with the expectation of regaining my former life, I soon discovered that re-integration into a "cancer-free" life was incredibly difficult. Previous work has shown that the inability to re-integrate can initiate feelings of isolation, anxiety, decreased self-esteem and depression which may be long-lasting without the proper support. ${ }^{[2]}$ An apparent flaw in our healthcare system is the lack of emotional support for this post-treatment barrier, forcing young adults to fight this battle alone. Due to my personal struggle with cancer, this article will focus on firsthand experiences with life after cancer, and where improvements are needed.

\section{Isolation - A Two Hit Mechanism}

Upon completing treatment and trying to re-integrate into my "old" life, the psychological struggle that was most difficult was overcoming social isolation. This isolation is one of the most devastating and yet understudied emotional trials for young adult cancer patients. ${ }^{[3]}$ In my opinion, this trial has the following origins: Firstly, a catalyst for isolation during treatment is navigating a medical system generally designed for a much older patient population. ${ }^{[2][4]}$ For example, the cancer centre where I was treated had an overwhelmingly older patient demographic with no form of peer-support tailored to young adults. It was a strange feeling to walk into a world renowned cancer centre and be one of the youngest people there by a minimum of thirty years. Although unacceptable, this is the standard throughout Canada, with only five centres having peersupport geared-towards young adults. ${ }^{[5]}$

Secondly, another origin of isolation comes posttreatment and is initiated by the inability to re-integrate into a "cancer-free" life. Due to my physical appearance and emotional instability, it was difficult to converse on a normal level with my peers which segregated me based on my disease instead of my age. This second origin of isolation compounded the first as the inability to converse with my peers affected personal relationships and academic endeavors, further preventing reintegration. Although it would seem that isolation would dissipate with time, the emotional long-term effects can influence patients well after their treatment has finished. ${ }^{[6]}$ As young adults have their entire lives ahead of them, emotional support networks need to be implemented to attenuate long term emotional damage due to isolation.

\section{Healthcare Program Implementation - What can we do?}

Based on the psychosocial challenges young adult patients face and the corresponding long term effects, it is critical that novel health care policies encompass these patient's post-treatment needs. Upon completing treatment, it was left up to me to go and find the support I needed which took strenuous searching to come up with the proper support. Finding the appropriate organizations is a very difficult task for survivors who, after finishing treatment, are fatigued or physically 
unable to make this commitment. In my opinion, this situation is unacceptable. Implementing young adult tailored support groups as part of post-treatment care should be mandatory and should be incorporated into all major centres. A way of supplementing a costly professionally-led system is to use a peer-led support system which has been shown to have few qualitative differences. ${ }^{[7]}$ By facilitating young adult survivors to create these groups, it allows both emotional support and encourages the formation of communities or "cancer families" which could further reduce the feeling of isolation. ${ }^{[8]}$ In either case, using professionally-led or peer-led support groups increases support for young adults which attenuates feelings of isolation and longterm associated distress.

\section{Conclusions - What is the next step?}

For the young adult cancer patient, the re-integration into a life after cancer is incredibly difficult. There is a significant need to create support systems to help bridge the young adult survivor's cancer life to their new "cancer-free" life. Healthcare support programs specific to young adults need to be placed in all major cancer centres throughout Canada. Only when these support systems are nationally accessible can we effectively help all young adult's psychological needs. By removing the onus of young adults to find their own support systems, their focus can shift from surviving to thriving.

\section{References}

1. E.E. Evan, L.K. Zeltzer. Psychosocial dimensions of cancer in adolescents and young adults, Cancer 107 (2006) 1663-1671.

2. C. Rabin, N. Simpson, K. Morrow, B. Pinto. Behavioral and psychosocial program needs of young adult cancer survivors, Qual. Health Res. (2010)

3. N.M. Aziz. Cancer survivorship research: State of knowledge, challenges and opportunities, Acta Oncol. 46 (2007) 417-432.

4. C.V. Fernandez, R.D. Barr. Adolescents and young adults with cancer: An orphaned population, Paediatr. Child. Health. 11 (2006) 103-106.

5. Canadian Cancer Society's Steering Committee. Canadian cancer statistics 2009, (2009) .

6. P. De, L.F. Ellison, R.D. Barr, R. Semenciw, L.D. Marrett, H.K. Weir, D. Dryer, E. Grunfeld. Canadian adolescents and young adults with cancer: Opportunity to improve coordination and level of care, CMAJ (2010) .

7. C. Stevinson, A. Lydon, Z. Amir. Characteristics of professionally-led and peer-led cancer support groups in the united kingdom, J. Cancer. Surviv. 4 (2010) 331-338.
8. J. Ussher, L. Kirsten, P. Butow, M. Sandoval. What do cancer support groups provide which other supportive relationships do not? the experience of peer support groups for people with cancer, Soc. Sci. Med. 62 (2006) 25652576.

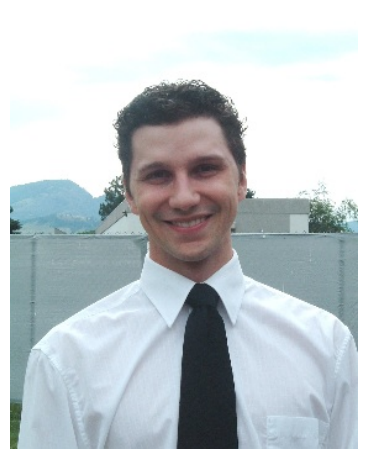

\section{Author Profile}

Timothy Buckland is currently working towards obtaining a MSc in Biochemistry at the University of Alberta. His research interests are focused on the Bcl-2 family of proteins and breast cancer therapy. As a cancer survivor he is also interested in young adult oncology and areas of improvement. 